\title{
QUANTIFICATIONAL DOMAINS AND RECURSIVE CONTEXTS
}

\author{
Barbara Partee \\ University of Massachusetts \\ Department of Linguistics \\ Amherst, MA 01003, USA \\ Internet: partee@cs.umass.edu
}

\begin{abstract}
The implicit delimiting or narrowing of the domain of quantification, e.g., in the case of "unselective quantifiers" such as the adverbs of quantification always, usually, mostly, etc., is a heavily contextdependent phenomenon that has much in common with anaphora, presupposition projection, the dynamics of reference time, reference location, etc., and other of the context-dependent phenomena discussed in Partee (1979). While many nonlinguistic factors clearly play a role in such phenomena, there are interesting issues at the intersection of discourse processing and sentence grammar, since in addition to context as constructed at the discourse level, there are subsentential "local contexts" which have limited lifespans and are constrained by aspects of sentence grammar, both syntactic and semantic.

So for example in the case of anaphora, while a pronoun can get its value from an entirely non-linguistic context, if the value of a pronoun is determined by a linguistic antecedent, there are grammatical contraints on the possible structural relations that may hold between antecedent and pronoun, as illustrated by the familiar "precede/command" conditions known since the early work of Ross and Langacker and illustrated in (1ab) below with respect to the possibility of interpreting "some people" as the antecedent of "they".
\end{abstract}

(1a) Some people complain loudly in the middle of the night and they make so much noise upstairs that one can't sleep.

(1b) They make so much noise upstairs that one can't sleep and some people complain loudly in the middle of the night.

In examples (2a-b) we see a similar restriction on the possibility of restricting the domain of the quantifier usually by means of material accessible in the linguistic context: and the relevant notion of accessibility turns out to be the same for the wide range of phenomena mentioned above. (2a) Henrik likes to travel. He goes to France in the summer and he usually travels by car. He goes to England for the spring holidays and he usually travels by ferry.

(2b) Henrik likes to travel. He usually travels by car and he goes to France in the summer. He usually travels by ferry and he goes to England for the spring holidays.

In the discourse (2b), unlike that in (2a), it is impossible to understand the domain of the quantifier usually to be limited to the trips to France and the trips to England on its two occurrences, so the discourse ends up sounding contradictory. This constraint on "backwards domain restriction" is analogous to constraints on backwards anaphora.

Similar constraints apply to the local satisfaction of presuppositions by virtue of material that has its source in the local linguistic context. And Heim has shown in her work on the presupposition projection problem that the relevant accessibility constraints are fundamentally semantic in nature, as can be seen from examples with propositional attitude verbs (which will be reviewed in the lecture) where examples with identical syntactic structure behave differently because of different presuppositional relationships among e.g., "belief worlds" and "hope worlds". Of course in many cases the semantic and syntactic structures are sufficiently parallel that the constraints can often be described either way.

The notions of topic and focus appear to be among the important linguistic notions that play a role in structuring these "recursive contexts"; recent work by Rooth and unpublished work by Von Fintel makes progress in relating focus structure to anaphoric structure more generally.

As Kempson has demonstrated, the same broad range of inferential processes that play a role in discourse anaphoric phenomena (e.g., in licensing the use of a definite article) also play a role in the corresponding phenomena when they show 
up in local subsentential contexts; so the fact that aspects of sentence grammar play a crucial role in defining accessibility relations for "antecedent" material in this whole family of phenomena does not mean that the phenomena themselves are to be described in sentence-grammar terms. One of the interesting issues, then, is the characterization of the nature of the interface between the grammatical and the extragrammatical mechanisms involved. Work by Sidner and Webber represents one early line of attack on related problems, and recent developments in dynamic semantics are another. This lecture will focus more on articulating the relationships among the different phenomena that appear to operate under common "accessibility" constraints than on choosing a particular formal approach to treating them. 